\title{
Evaluación de los patrones alimentarios y la nutrición en cuatro comunidades rurales
}

Judith Aguirre-A renas, Lic. en N utr., ${ }^{(1)}$ Margarita Escobar-Pérez, Téc. en N utr., ${ }^{(1)}$

Adolfo Chávez-Villasana, M.C., M. P. H.(1)

\begin{abstract}
Aguirre-Arenas J, Escobar-Pérez $M$, Chávez-Villasana A.

Evaluación de los patrones alimentarios y la nutrición en cuatro comunidades rurales. Salud Publica Mex 1998;40:398-407.
\end{abstract}

\section{Resumen}

O bjetivo. Estudiar el efecto del cambio agrícola y del subsecuente cambio social sobre la situación nutricional en cuatro comunidades de diferentes regiones y climas de México, las que desde el inicio de los años setenta cambiaron la agricultura tradicional de maíz por el cultivo comercial de sorgo. Material y métodos. Se volvió a aplicar una encuesta dietética y nutricional (1996) para evaluar el cambio en hábitos de consumo y el estado de nutrición de los niños de cuatro comunidades que habían sido estudiadas 12 años antes (1984). Resultados. La alimentación continuó diversificándose con mejor calidad proteica, el consumo de alimentos industrializados aumentó y el de maíz bajó. En promedio el estado nutricional de los niños mejoró, a pesar de que desde el punto de vista agrícola el cultivo comercial de sorgo entró en crisis y las comunidades han vuelto parcialmente a cultivar maíz, a diversificar la producción 0 a abandonarla migrando. Conclusiones. A pesar de, primero, haber dejado el cultivo tradicional de maíz y, después, de haber tenido problemas con el cultivo comercial de sorgo, las comunidades han seguido mejorando sus condiciones nutricionales. No obstante, dicha mejoría se debe, sobre todo, a su capacidad para llevar a la práctica estrategias de so brevivencia, a la ayuda económica que les brinda la población que emigra y a una mejor disponibilidad alimentaria.

Palabras clave: conducta alimentaria; estado nutricional; asentamientos rurales; indicadores econó micos; antro pometría; México

\section{A guirre-Arenas J, Escobar-Pérez $M$, Chávez-Villasana A. \\ Evaluation of the food consumption and nutrition in 4 rural communities. \\ Salud Publica Mex 1998;40:398-407.}

\section{A bstract}

Objective. To study the effect of agricultural change and subsequent social change on the nutritional situation of 4 communities of different regions and climates, that since the 70's have changed from traditional corn production to commercial sorgum production. Material and methods. A new dietetic and clinical survey (1996) was performed to follow-up the changes in the 4 communities studied 12 years before (1984). Results. The food consumption pattern continued changing, with more dietary diversification, better protein quality and less corn consumption. The child nutrition status on average was better. This happened in spite of the fact that the sorgum production was in a crisis and the communities partially returned to corn production, diversified production or migrated from the communities. Conclusions. In spite of first abandoning traditional corn production and later having problems with the commercial product (sorgum), the communities are still improving their nutrition. This is mostly due to implementing certain survival strategies, to receiving support from the people from the community who migrated and to a better food availability.

Key words: feeding behavior; nutritional status; rural settlements; economic indexes; anthropometry; Mexico

(1) Departamento de Educación N utricional, Subdirección General de N utrición de Comunidad, Instituto N acional de la N utrición Salvador Zubirán, México.

Fecha de recibido: 30 de junio de 1997 • Fecha de aprobado: 13 de julio de 1998

Solicitud de sobretiros: Lic. Judith A guirre Arenas. Departamento de Educación Nutricional, Subdirección General de Nutrición, Instituto Nacional de la N utrición Salvador Zubirán. Vasco de Q uiroga 15, Tlalpan, 14000 México, D.F., México. 
$\mathrm{V}$ arios expertos agrícolas mexicanos y extranjeros, interesados en el desarrollo socioeconómico, han mantenido un debate acerca de las consecuencias de la modernización agrícola sobre la alimentación y la nutrición de las comunidades, en particular cuando se cambia del modelo productivo de la autosuficiencia tradicional a la producción comercial. ${ }^{1}$ Por una parte, existen estudios que muestran que el cambio altera la autosuficiencia, la cadena alimentaria, la estructura social productiva y el equilibrio dietético, lo que a su vez da lugar a una agudización de los problemas de desnutrición ${ }^{2}$ pero, por otra parte, también hay datos que muestran lo contrario, es decir, que la agricultura comercial puede dar lugar a un acceso más variado a los alimentos y a una mejor nutrición. ${ }^{3}$

En el Instituto Nacional de la Nutrición Salvador Zubirán (INNSZ) existen algunas experiencias previas que señalan que, en general, el abandono del monocultivo de maíz es positivo, aunque en los años inmediatos al cambio a menudo sucede que las familias con menos recursos y menor potencial de trabajo conservan un bajo nivel alimentario y que sus niños presentan desnutrición. ${ }^{4}$ Una situación bastante frecuente es que las comunidades que transforman su modelo productivo también cambian en otros aspectos, tendiendo más a acudir a los comercios y a disfrutar de mejores servicios, y sufriendo un cambio cultural importante, con consecuencias positivas y negativas. ${ }^{5}$

El presente trabajo se planeó como una segunda visita a cuatro comunidades de diferentes regiones, climas y áreas culturales: Quebrantadero, Morelos; Guía del Porvenir, Tamaulipas; Derramadero, San Luis Potosí, y Bateas Alcalde, en Michoacán. En 1984 se seleccionaron para un estudio pues se sabía que, desde los inicios de los años setenta, habían comenzado a sustituir progresivamente el cultivo tradicional de maíz destinado al consumo por un nuevo cultivo, el de sorgo, que fue promovido por algunas compañías productoras de forraje. En el estudio de 1984 el objetivo era determinar si el cambio de un cultivo tradicional y básico para la alimentación, por un cultivo comercial, habría afectado la nutrición de la población. Los resultados de 1984 no dieron lugar a conclusiones definitivas, sobre todo porque no se tenían datos previos de las comunidades mismas. Sin embargo, al comparar la información con la de otros estudios de comunidades semejantes ubicadas en las mismas zonas y que no cambiaron su modelo productivo, se pudo observar que las cuatro comunidades se encontraban en una situación ligeramente mejor. No obstante, esa mejoría se atribuyó más a cambios en las condiciones culturales y sociales que al cambio agrícola, sobre todo porque

en las cuatro comunidades se iniciaron a la vez algunas fuentes de empleo alternativo que pudieron haber ayudado en forma más decisiva que los cambios agrícolas. ${ }^{6,7}$

En el estudio de 1996 se observó, desde la perspectiva económica, una severa crisis agrícola -sobre todo en el cultivo del sorgo- que se presentó como consecuencia de la crisis económica general del país, agudizada por el Tratado de Libre Comercio de América del Norte (TLCAN) y por la libre entrada de granos de importación. En 1996 en las comunidades había un escaso interés en la agricultura aunque seguían produciendo sorgo y habían vuelto, parcialmente, a la producción de maíz para consumo, sobre todo en la comunidad perteneciente a Tamaulipas. Muchos adultos, hombres y mujeres, habían emigrado a Estados Unidos de América (EUA) o se habían trasladado a los centros urbanos de México; además, habían recurrido a varias estrategias de sobrevivencia como las ventas ambulantes.

El fenómeno más notable en todas las comunidades fue el de las migraciones, tanto las temporales, con el regreso anual de los trabajadores trayendo recursos adicionales, como las más permanentes; en este último caso los que se han ido, sobre todo a EUA, envían dinero e inclusive despensas de alimentos a sus familias.

El objetivo principal de este estudio fue comparar los niveles de consumo de alimentos y el estado de nutrición con los registrados 12 años antes, para conocer las consecuencias, en un plazo más largo, del cambio agrícola y también de las nuevas condiciones sociales, tales como una economía más comercial, la presencia de factores de crisis y las nuevas estrategias de sobrevivencia.

\section{Material y métodos}

Se partió de la metodología del estudio previo ${ }^{6}$ para establecer los lineamientos de la investigación presente. La muestra se determinó por medio de un muestreo aleatorio simple, seleccionando 40 familias de cada comunidad, a partir del listado hecho durante el Censo General de Población de 1990.

Se comparó la información dietética y nutricional recabada en dos estudios transversales descriptivos, llevados a cabo en las mismas estaciones de los años 1984 y 1996, en cuatro comunidades rurales. Estas últimas fueron seleccionadas en 1984 de acuerdo con el criterio principal de que tuvieran diferente ecología, tamaño, tenencia de la tierra y capacidad de acceso a los mercados. Dos de las comunidades están situadas 
en el norte; una, Derramadero, en la zona árida de San Luis Potosí, y la otra, Guía del Porvenir, en una zona de riego del estado fronterizo de Tamaulipas; otra comunidad, Bateas-Alcalde, está situada en Michoacán, en la llamada Tierra Caliente, semiárida pero con posibilidad de riego, y la última, Quebrantadero, en el centro, en el estado de Morelos, cerca de la Ciudad de México. Todas tuvieron incrementos semejantes en la producción de sorgo durante los años anteriores y decrementos en la producción de maíz. También se consideró el hecho de que no tuvieran programas de desarrollo especiales y que los ejidos y sus líderes cooperaran con el programa.

Las comunidades Guía del Porvenir, Derramadero y Bateas Alcalde tenían alrededor de 1000 habitantes, y Quebrantadero, cerca de 3 000. Las tres primeras tenían ejidos con parcelas familiares y estaban constituidas por alrededor de 150 familias, mientras que Quebrantadero contaba con 84. Dos de los ejidos tenían riego y en todos se había introducido el sorgo después de 1970, o sea por lo menos 14 años antes de la primera visita y 27 antes de la actual. Desde 1984 en todos los ejidos existían algunas características de modernización como, por ejemplo, los tractores, situación que no ha cambiado en los últimos 13 años debido, posiblemente, a la crisis y a la falta de tecnología y de inversiones.

La información dietética de los dos estudios se obtuvo por medio de la técnica de encuesta de recordatorio de 24 horas, ${ }^{8}$ que registra las cantidades de alimentos consumidos el día inmediato anterior, desglosadas en los tiempos de comida habituales para cada comunidad, complementado con ciertos lineamientos de la técnica de pesas y medidas. Debido a las variaciones regionales, tanto del alimento (piezas de pan, manojos de hojas verdes, etc.) como de las diferentes medidas que regionalmente se usan y que no siempre son iguales, como el "almud" de maíz, la "sardina" de frijol y las tazas de bebidas regionales, se utilizó una báscula dietética marca Terrallon, con precisión de 2 gr, así como cucharas y tazas graduadas para medir las porciones de alimentos. Se recabó información sobre alimentos consumidos fuera del hogar, es decir, acerca de las preparaciones (ingredientes, cantidades y técnicas de preparación) consumidas en la misma comunidad o fuera de ella; de este modo, se compraron los alimentos necesarios y se pidió a las señoras que los prepararan según sus costumbres, para después pesarlos y obtener promedios.

Los datos obtenidos fueron transformados a gramos, de acuerdo con los listados de pesos promedio de alimentos por comunidad, y para conocer su valor nutritivo se calculó el consumo de nutrimentos de acuerdo con las Tablas de valor nutritivo de los alimentos de mayor consumo en México. ${ }^{9}$ Su nivel de adecuación se estimó comparándolos con las recomendaciones nutricionales para México del INNSZ.

Una misma persona, previamente estandarizada en las técnicas antropométricas según el protocolo de Habicht, ${ }^{10}$ tomó el peso y la talla de los miembros mayores de dos años de las familias encuestadas, con los individuos descalzos, utilizando una báscula electrónica digital marca SECA ALPHA de $150 \mathrm{~kg}$ y un estadímetro Microtoise de $200 \mathrm{~cm}$. Se siguió la metodología de acuerdo con la población de referencia recomendada por el National Center for Health Statistics-Organización Mundial de la Salud..$^{11}$ A los niños menores de dos años se les tomó peso en kilogramos y longitud supina en centímetros por medio de una báscula de "reloj" marca Jacobs Detecto y un infantómetro de madera con precisión de $1 \mathrm{~mm}$ para la longitud.

En el presente trabajo se muestran los datos correspondientes a 259 prescolares de 1984 y a 266 de 1996; se clasificó su estado nutricional de acuerdo con el puntaje $z$, según las categorías de la Organización Mundial de la Salud..$^{11}$ Los resultados se comparan por medio de la prueba $t$ de Student $(p=0.5)$.

Un equipo de trabajo compuesto por cuatro antropólogos sociales -que vivieron un mes en cada una de las comunidades- y tres nutriólogos especialmente capacitados recolectó los datos sobre los aspectos sociales y dietéticos. Posteriormente, la información se capturó en archivos con formato DBF y se procesó por medio de programas de computación diseñados en el INNSZ. Los resultados descritos en el presente artículo se refieren, exclusivamente, a las familias de las cuatro comunidades estudiadas.

\section{Resultados}

\section{Características socioeconómicas}

Respecto a la tenencia de la tierra, $67,69.7$ y $75 \%$, de las familias estudiadas tienen terrenos ejidales en las comunidades pertenecientes a Morelos, Tamaulipas y San Luis Potosí, respectivamente, y 35.5\%, en Michoacán. Alrededor de 30\% de las familias de las cuatro comunidades carecen de tierras pero, en general, logran que se les arrienden o les presten tierras para cultivos. La tenencia de la tierra varía entre las comunidades: en Quebrantadero, 21\% de las familias tiene acceso a menos de tres hectáreas, en tanto que en Guía del Porvenir $71 \%$ tiene acceso a 20 hectáreas o más; en Derramadero $18 \%$ tiene menos de tres hectáreas, y ninguna 
familia tiene más de ocho hectáreas. En Bateas Alcalde $6 \%$ tiene menos de tres hectáreas, y $16 \%$, más de 12 hectáreas.

Durante los años setenta y al inicio de los ochenta, las familias de Bateas Alcalde y Guía del Porvenir obtenían la mayor parte de su ingreso de la agricultura; en cambio, en Quebrantadero y en Derramadero una buena parte del ingreso provenía ya de salarios por trabajos realizados fuera de la comunidad. En el caso de Derramadero, en la encuesta de 1984 se encontró que $80 \%$ ya había trabajado en EUA y $43 \%$ tenía, cuando menos, un hijo en aquel país.

En 1984 el ingreso monetario de las familias estudiadas en Derramadero y Bateas Alcalde era bastante menor que el de las de Guía del Porvenir y Quebrantadero. En todas se encontraron importantes diferencias entre las familias, ya que algunas tenían un ingreso mucho más alto que las otras. En la encuesta de 1996 se observó que los ingresos provenientes de la agricultura disminuyeron en $24 \%$ para las familias de Guía del Porvenir, en 3.7\% para las de Derramadero y en 52.2\% para las de Bateas Alcalde. En cambio, en Quebrantadero dicho ingreso aumentó en $49.1 \%$.

En la encuesta de 1996 el maíz había vuelto a ser el principal producto agrícola en todas las comunidades, y el sorgo aparecía en segundo lugar; así, en Quebrantadero representó $17.9 \%$ del producto agrícola; en Derramadero, $24.1 \%$, y en Bateas Alcalde, 28.6\%. En la comunidad de Guía del Porvenir, ninguna familia de las encuestadas sembró sorgo. En Bateas Alcalde 18\% de las familias ha vuelto a producir ajonjolí, un antiguo cultivo local. En todas las comunidades, entre 20 y $40 \%$ de las familias no siembran nada. En pequeña escala comienzan a sembrar cebolla, caña y pepino. En Guía del Porvenir y Bateas Alcalde han dejado mucha tierra como pastizal para el ganado.

\section{Consumo de alimentos}

Las encuestas que se hicieron antes de 1970 en esas regiones tendían a detectar una alimentación insuficiente, monótona y desequilibrada en sus nutrimentos, porque consistía en un consumo elevado de maíz -entre 50 y $80 \%$ de la energía-, al que la población agregaba cantidades variables de frijol, chile, algunas verduras y rara vez otros alimentos. Era de baja densidad energética, sobre todo por el escaso consumo de grasa. Era también de pobre calidad proteica y baja en la disponibilidad de micronutrimentos como el hierro y de vitamina $\mathrm{A}$, ácido ascórbico, niacina y riboflavina. ${ }^{12}$

En las encuestas de 1984 hechas en las cuatro comunidades se observaron patrones alimentarios más diversificados, aunque la dieta seguía basándose en maíz y frijol, entonces complementada con diversas verduras, frutas y alimentos de origen animal. Agregaban también algunos alimentos industrializados basados en azúcares y grasas, además de pastas para sopa, pan, galletas de bajo costo y refrescos. Todo eso proporcionaba una alimentación escasa en cantidad y mala en calidad, pero de todas maneras ya más abundante y diversificada, sobre todo en las épocas de trabajo agrícola, cuando antes se encontraba una especial reducción en los niveles de consumo.

La información que se obtuvo en la encuesta de 1996 indicaba cambios bastante marcados en los patrones alimentarios; el consumo de cereales y otros granos fue menor, y el de otros alimentos, mayor. Se encontraron formas diferentes respecto a su adquisición y preparación. El maíz continuó siendo el alimento básico en las cuatro poblaciones; sin embargo, su consumo había disminuido en cantidad, hecho que se hace más evidente al analizar el total del maíz consumido (cuadro I). En la última encuesta se observó que el maíz se adquiría también en forma de harina nixtamalizada con la que fácilmente se preparan las tortillas, o bien, que estas últimas eran compradas por las familias en los expendios de la comunidad, donde se elaboraban en máquinas. En Guía del Porvenir y Bateas Alcalde también consumían tortillas de harina de trigo o mezclaban las harinas de maíz con trigo durante su preparación. En general se observó una disminución en el consumo de productos de trigo en Guía del Porvenir y Derramadero, sobre todo por una reducción en las compras de grano y de pan. Las otras formas industrializadas de trigo, como pastas para sopa y galletas, se mantuvieron, ya que eran consumidas con cierta constancia por varias familias. El arroz mostró un ligero aumento en todas las comunidades.

En el cuadro II se presentan las cifras correspondientes al consumo del resto de los alimentos; los cambios más evidentes fueron en los frijoles y, sobre todo, en las verduras, cuyo consumo se duplicó en las cuatro comunidades. Respecto a la variedad de estas últimas, se notifican, además del jitomate, la cebolla y el chile-que son todavía frecuentes y forman parte de la mayoría de los platillos preparados-, calabacitas, ejotes, zanahoria, chayotes, pepinos, aguacate, otros tipos de chile, papas, lechuga, col y nopales. Las frutas que más se consumieron fueron, sobre todo, las de temporada, de acuerdo con las diferentes comunidades, como mangos, tunas, plátanos, naranjas, guayaba, papaya, melón, sandía y ciruelas.

Los productos de origen animal son básicamente la leche y los huevos; el consumo de queso fresco preparado en las mismas comunidades es bastante frecuente, y en todos se observa un incremento, con excepción 


\section{Cuadro I}

\section{Cambios en el consumo diario de Cereales Per CÁpita.}

\section{Gramos de peso bruto según adquisición y preparación. MéXico, 1984 y 1996}

\begin{tabular}{|c|c|c|c|c|c|c|c|c|}
\hline Alimentos & Quebran & Morelos & Guía del $\mathrm{F}$ & Tamaulipas & Derramad & Luis Potosí & Bateas A & Michoacán \\
\hline & 1984 & 1996 & 1984 & 1996 & 1984 & 1996 & 1984 & 1996 \\
\hline Maíz (grano) & 387 & 35 & 242 & 31 & 357 & 78 & 387 & 81 \\
\hline Tortillas de maíz (compradas) & 0 & 287 & 0 & 165 & 0 & 129 & 0 & 82 \\
\hline Harina de maíz & 0 & 0 & 0 & 42 & 0 & 102 & 0 & 119 \\
\hline Hojuelas y maicena & 0 & 2 & 0 & 1 & 0 & 2 & 0 & 1 \\
\hline Total de maíz & 387 & 233 & 242 & 194 & 357 & 285 & 387 & 276 \\
\hline Trigo (grano) & 0 & 0 & 33 & 0 & 3 & 0 & 3 & 0 \\
\hline Harina de trigo & 0 & 1 & 4 & 39 & 0 & 5 & 2 & 42 \\
\hline Pan blanco y dulce & 30 & 30 & 85 & 13 & 44 & 11 & 30 & 8 \\
\hline Pastas & 6 & 7 & 13 & 11 & 13 & 12 & 6 & 9 \\
\hline Pastelillos & 0 & 1 & 0 & 0 & 0 & 5 & 0 & 3 \\
\hline Galletas & 0 & 4 & 0 & 4 & 0 & 6 & 0 & 9 \\
\hline Total de trigo & 21 & 28 & 92 & 60 & 38 & 31 & 26 & 66 \\
\hline Arroz & 9 & 11 & 19 & 25 & 11 & 20 & 9 & 18 \\
\hline
\end{tabular}

Cuadro II

Cambios en el consumo diario de alimentos Per CÁPITA En MiliLitros y gramos de Peso bruto. MÉxıCO, 1984 Y 1996

\begin{tabular}{|c|c|c|}
\hline Alimentos & Quebrantadero, Morelos & Guía del Porvenir,Tamaulipas \\
\hline & $1984 \quad 1996$ & $1984 \quad 1996$ \\
\hline
\end{tabular}

\begin{tabular}{lrrrrrrrrr} 
Leche y queso & 75 & 261 & 175 & 71 & 146 & 149 & 75 & 124 \\
\hline Carnes & 51 & 66 & 70 & 72 & 33 & 44 & 51 & 49 \\
\hline Huevo & 16 & 39 & 48 & 37 & 18 & 48 & 16 & 19 \\
\hline Frijoles & 27 & 60 & 31 & 71 & 36 & 62 & 27 & 54 \\
\hline Frutas & 45 & 178 & 163 & 142 & 88 & 88 & 45 & 78 \\
\hline Verduras & 55 & 204 & 96 & 121 & 87 & 106 & 55 & 143 \\
\hline Raíces feculentas & 11 & 15 & 37 & 33 & 18 & 36 & 11 & 49 \\
\hline Grasas para cocinar & 19 & 37 & 51 & 40 & 21 & 34 & 19 & 30 \\
\hline Azúcares & 23 & 42 & 56 & 34 & 33 & 33 & 23 & 22 \\
\hline Refrescos & 0 & 37 & 251 & 238 & 63 & 250 & 69 & 167 \\
\hline Bebidas alcohólicas & 0 & 1 & 0 & 0 & 0 & 23 & 0 & 9
\end{tabular}

Alimentos industrializados

$\begin{array}{lllllllll}\text { Alimentos de origen animal* } & 0 & 30 & 0 & 25 & 0 & 29 & 0 & 47\end{array}$

$\begin{array}{lllllllll}\text { tros }^{\ddagger} & 0 & 15 & 0 & 12 & 0 & 11 & 0 & 4\end{array}$

* Jamón, salchichas, chorizo, pollo rostizado, atún enlatado, leche en polvo, crema y yogur

₹C afé instantáneo, chocolate en polvo, consomé, mayonesa y gelatina 
de Guía del Porvenir. La carne más consumida es la de pollo y un poco menos la de res; la carne de cerdo no es de consumo habitual. En Guía del Porvenir el consumo de pescado se registró en mayor cantidad, en variedades como la tilapia, el bagre y la mojarra.

El consumo de grasas para cocinar en gramos de peso bruto, sobre todo de manteca de cerdo, aumentó en $48 \%$ en Quebrantadero, y 38 y 37\% en las comunidades de Derramadero y Bateas Alcalde, respectivamente. Las familias encuestadas en Guía del Porvenir y Bateas Alcalde utilizaron manteca vegetal en la preparación de tortillas de harina de trigo; sólo en Guía del Porvenir disminuyeron los consumos de grasas y azúcares.

Los cambios en los patrones alimentarios de estas poblaciones son más evidentes respecto a los productos industrializados: así está el café soluble, que, en Quebrantadero, Derramadero y Guía del Porvenir, lo consumen aproximadamente $80 \%$ de las familias, y en donde ha desplazado al consumo del café en grano y también al té. Las cantidades de refrescos embotellados, sobre todo los de cola, aumentaron en todas las comunidades excepto en Quebrantadero. Asimismo, consumieron polvo para refrescos del tipo Tang o Kool-Aid, que preparan en forma de congelados (paletas) y son consumidos principalmente por los niños. Respecto a las bebidas alcohólicas, el consumo fue menor comparado con las cifras indicadas en otros estudios:13,14 $106 \mathrm{ml}$ per cápita diarios en adultos en
1981, y 3.51 per cápita anuales en 1996. Lo anterior posiblemente se explica porque la técnica utilizada en esta encuesta sólo registra lo consumido en el hogar.

Por otra parte, también se notificaron consumos de pequeñas cantidades de cereales empacados, carnes frías (salchichas, jamón y chorizo), pollo rostizado, chocolates solubles, jugos y atún enlatados. Las leches en polvo se destinan, sobre todo, a los lactantes o a los escolares. Otros productos muy demandados son los chiles enlatados, los fritos (papitas y chicharrón de harina), las verduras y las sopas enlatadas.

En Derramadero y Guía del Porvenir algunas familias recibían despensas de familiares que trabajan en EUA. En las cuatro comunidades estudiadas había programas de alimentación, tanto gubernamentales como privados, que repartían algunos productos como harina de maíz, arroz, azúcar, atún, sardinas, frijol, leche y chocolate en polvo, galletas, maicena de sabores y gelatinas. Estos son proporcionados cada mes a los niños becados por la Secretaría de Educación Pública y mediante los desayunos escolares que se reparten diariamente en los jardines de niños y en las primarias.

\section{Consumo de nutrimentos}

Los consumos de calorías y proteínas en la encuesta de 1996 (cuadro III) fueron adecuados y un poco más altos en proteínas que los encontrados en el estudio de

Cuadro III

\section{Cambios en el consumo diario de nUtrimentos Per CÁpita. México, 1984 y 1996}

\begin{tabular}{|c|c|c|c|c|c|c|c|c|c|}
\hline \multirow[t]{2}{*}{ Alimentos } & & \multicolumn{2}{|c|}{ Quebrantadero, M orelos } & \multicolumn{2}{|c|}{ Guía del Porvenir,Tamaulipas } & \multicolumn{2}{|c|}{ Derramadero, San Luis Potosí } & \multicolumn{2}{|c|}{ Bateas Alcalde, Michoacán } \\
\hline & & 1984 & 1996 & 1984 & 1996 & 1984 & 1996 & 1984 & 1996 \\
\hline Energía & kcal & 2000 & 2255 & 2476 & 2204 & 2124 & 2256 & 2000 & 2179 \\
\hline \% de adecuación* & & 67.6 & 100.0 & 85.3 & 91.8 & 79.1 & 100.1 & 67.6 & 96.2 \\
\hline Proteínas & g & 48.4 & 69.1 & 55.5 & 62.5 & 53.0 & 61.2 & 48.4 & 60.6 \\
\hline \% de adecuación* & & 63.8 & 131.5 & 70.2 & 112.1 & 70.3 & 114.3 & 63.8 & 115.9 \\
\hline Hierro & $\mathrm{mg}$ & 12.4 & 21.1 & 12.8 & 17.3 & 13.1 & 18.8 & 12.4 & 16.7 \\
\hline Vitamina A & $\mathrm{mcg}$ & 210 & 998 & 467 & 502 & 347 & 506 & 210 & 511 \\
\hline Vitamina C & $\mathrm{mg}$ & 20 & 110 & 54 & 40 & 30 & 43 & 20 & 50 \\
\hline $\bar{N}$ iacina & $\mathrm{mg}$ & 10.0 & 10.8 & 9.8 & 8.9 & 9.6 & 9.9 & 10.0 & 10.4 \\
\hline Riboflavina & $\mathrm{mg}$ & 0.5 & 1.3 & 0.8 & 0.9 & 0.7 & 0.9 & 0.5 & 0.8 \\
\hline Tiamina & $\mathrm{mg}$ & 1.8 & 1.5 & 1.7 & 1.4 & 1.8 & 1.5 & 1.8 & 1.4 \\
\hline Calcio & $\mathrm{mg}$ & 789 & 1409 & 815 & 868 & 969 & 975 & 789 & 889 \\
\hline Sodio & $\mathrm{mg}$ & 301 & 584 & 831 & 360 & 443 & 512 & 301 & 298 \\
\hline Zinc & $\mathrm{mg}$ & 1.9 & 4.8 & 3.2 & 5.2 & 2.3 & 7.1 & 1.9 & 6.3 \\
\hline Grasas totales & $\mathrm{g}$ & 48.3 & 79.7 & 85.0 & 72.3 & 51.1 & 64.5 & 48.3 & 63.6 \\
\hline Grasas saturadas & $g$ & 10.9 & 18.5 & 23.1 & 14.0 & 12.0 & 17.5 & 10.9 & 18.7 \\
\hline Colesterol & $\mathrm{mg}$ & 125 & 286 & 324 & 240 & 135 & 300 & 125 & 162 \\
\hline Fibra & g & 47.1 & 19.0 & 34.4 & 19.8 & 45.8 & 27.9 & 47.1 & 29.1 \\
\hline
\end{tabular}

*Adecuación de consumos de acuerdo con la composición de la población por edad y sexo en ambos estudios 
1984. El consumo de calcio aumentó, sobre todo en las comunidades pertenecientes a Morelos y Michoacán debido, probablemente, a una mayor ingestión de nopales y de tortillas. Las cantidades de vitamina A y C aumentaron en todas las comunidades en el segundo estudio; sin embargo, las cifras aún son inferiores a las recomendaciones diarias per cápita para adultos. ${ }^{9} \mathrm{La}$ comunidad situada en Tamaulipas presentó cifras más bajas.

El consumo de colesterol se elevó en forma importante, aunque se encuentra dentro de las recomendaciones diarias para adultos (menos de $300 \mathrm{mg}$ ), excepto en Derramadero. Las cifras de riboflavina prácticamente no tuvieron cambios y continúan siendo inferiores a las recomendaciones diarias. El consumo de nutrimentos fue más elevado en Quebrantadero, quizá porque presenta características físicas y económicas más de tipo urbano que rural.

En el cuadro IV se muestra cómo el aporte de energía de la dieta consumida en 1984 correspondía, en mayor medida, a los cereales y derivados. En la segunda evaluación, dicho aporte disminuyó en las cuatro comunidades, y en Guía del Porvenir, la disminución fue más acentuada. Estos cambios se debieron a un mayor consumo de frijol, frutas y verduras, alimentos de origen animal, refrescos y alimentos industrializados, esto es, a una diversificación, lo que dio lugar a una alimentación con tendencia a parecerse a la de las poblaciones urbanas.

En cuanto al origen de las proteínas, se observa un cambio, ya que aumentaron las de origen animal y disminuyeron los alimentos vegetales. En Quebrantadero las proteínas animales representaron $41.6 \%$ del consumo proteico, mientras que en las demás comunidades fue de alrededor de $30 \%$. En tres de las comunidades aumentó la participación de los alimentos de origen animal en la dieta, con excepción de la de Guía del Porvenir, en la que se sostuvieron aproximadamente los mismo valores; se observa, además, que en esa misma comunidad y Bateas Alcalde los alimentos industrializados representaron un mayor porcentaje en comparación con el estudio inicial.

\section{Estado de nutrición de los niños}

La prevalencia de desnutrición en los niños (cuadro V) según el indicador peso para la edad bajó de $27.5 \%$ en 1984 a 13\% en 1996 en Quebrantadero; de 26.4 a 9.1\% en Guía del Porvenir; de 38.7 a $25.6 \%$ en Derramadero, y de 56.4 a $26.6 \%$ en Bateas Alcalde.

En general, el sexo masculino fue el más beneficiado por los cambios sociales del periodo analizado, ya que la prevalencia de desnutrición fue más alta en las niñas, con $28.3 \%$ en Derramadero y $26.3 \%$ en Bateas Alcalde; en Quebrantadero y Guía del Porvenir fue de 15.6 y $13 \%$, respectivamente. Por otra parte, los casos de sobrepeso y obesidad se presentaron con mayor frecuencia en los niños que en las niñas.

\section{Discusión}

La selección de las comunidades estudiadas se basó en el hecho de que, al iniciarse la década de los años setenta, cambiaron su patrón productivo tradicional de maíz por el de tipo comercial de sorgo. En la encuesta de 1996 se encontraron dos cambios más: las comu-

\section{Cuadro IV}

\section{Cambios en la distribución porcentual de calorías según su origen. México, 1984 y 1996}

\begin{tabular}{|c|c|c|c|c|c|c|c|c|}
\hline \multirow[t]{2}{*}{ Alimentos } & \multicolumn{2}{|c|}{ Quebrantadero, M orelos } & \multicolumn{2}{|c|}{ Guía del Porvenir,Tamaulipas } & \multicolumn{2}{|c|}{ Derramadero, San Luis Potosí } & \multicolumn{2}{|c|}{ Bateas Alcalde, Michoacán } \\
\hline & 1984 & 1996 & 1984 & 1996 & 1984 & 1996 & 1984 & 1996 \\
\hline Cereales y derivados & 72.3 & 41.3 & 48.9 & 35.9 & 67.1 & 49.3 & 72.3 & 46.7 \\
\hline Leguminosas y oleaginosas & 4.5 & 9.7 & 4.1 & 11.2 & 5.6 & 9.9 & 4.5 & 9.1 \\
\hline Frutas, verduras y raíces feculenta & $\operatorname{tas} 2.0$ & 5.6 & 4.2 & 3.4 & 2.9 & 3.7 & 2.0 & 4.2 \\
\hline Grasas para cocinar & 8.3 & 14.4 & 17.9 & 14.8 & 8.6 & 9.0 & 8.3 & 12.3 \\
\hline Azúcares & 4.4 & 6.5 & 8.7 & 6.1 & 5.9 & 5.2 & 4.4 & 3.1 \\
\hline Leche y derivados & 2.5 & 9.4 & 4.7 & 5.4 & 4.9 & 5.0 & 2.5 & 4.5 \\
\hline Carnes y derivados & 3.9 & 4.5 & 4.3 & 5.2 & 2.4 & 3.4 & 3.9 & 2.5 \\
\hline Huevos & 1.1 & 2.2 & 2.7 & 2.4 & 1.2 & 3.1 & 1.1 & 1.3 \\
\hline Alimentos industrializados & 0.0 & 5.5 & 0.0 & 10.8 & 0.0 & 6.5 & 0.0 & 13.2 \\
\hline Refrescos & 1.0 & 0.9 & 4.5 & 4.8 & 1.4 & 4.9 & 1.0 & 3.1 \\
\hline O rigen animal & 7.5 & 18.9 & 11.7 & 14.3 & 8.5 & 14.5 & 7.5 & 11.8 \\
\hline O rigen vegetal & 92.5 & 81.1 & 88.3 & 85.7 & 91.5 & 85.5 & 92.5 & 88.2 \\
\hline
\end{tabular}




\section{Cuadro V \\ Cambios en la prevalencia de desnutrición según el indicador peso/edad en cuatro comunidades rurales. Méxıco, 1984 y 1996}

\begin{tabular}{|c|c|c|c|c|c|}
\hline \multirow[b]{3}{*}{ Comunidades } & \multicolumn{4}{|c|}{ Desnutrición (> $1 \mathrm{DE}, \mathrm{P} / \mathrm{E}$ ) } & \multirow{3}{*}{$\begin{array}{l}\text { Nivel de significación } \\
\text { de diferencia }\end{array}$} \\
\hline & \multicolumn{2}{|c|}{1984} & \multicolumn{2}{|c|}{1996} & \\
\hline & Casos & $\%$ & Casos & $\%$ & \\
\hline Q uebrantadero, Morelos & 19 & 27.5 & 6 & 13.0 & 0.05 \\
\hline Guía del Porvenir,Tamaulipas & 14 & 26.4 & 4 & 9.1 & 0.05 \\
\hline Derramadero, San Luis Potosí & 22 & 38.7 & 20 & 25.6 & 0.05 \\
\hline Bateas Alcalde, Michoacán & 45 & 56.4 & 26 & 26.6 & 0.01 \\
\hline Total de niños & 100 & 38.6 & 56 & 21 & 0.01 \\
\hline
\end{tabular}

nidades regresaron parcialmente al cultivo de maíz y comenzaron a intentar la producción de algunos otros alimentos como el ajonjolí y el pepino en Bateas Alcalde y, en menor escala, cebolla y caña en la de Quebrantadero. Esos cambios estuvieron aparejados con otros de tipo social, sobre todo por crecimientos de carácter poblacional y comercial, así como por la ampliación de las comunicaciones y de varios servicios como los educativos y los de salud.

En los aspectos propiamente de salud y nutrición, en 1996 se encontró que todas las comunidades estudiadas ya tenían centros de salud y escuelas no sólo primarias, sino también telesecundarias. En Derramadero había un programa comunitario de atención médica familiar con clínica en la cabecera municipal; ahí también existen grupos organizados de voluntarios que trabajan en diversas áreas: salud, nutrición y manejo de ayudas en alimentos que se otorgan por medio de becas a los escolares y mediante desayunos a prescolares. En la última visita se observó que había, además de la agricultura, nuevas fuentes de empleo: ventas ambulantes, producción de artesanías y prestación de servicios como el planchado de ropa; destacó, sobre todo, la búsqueda de trabajos fuera de la comunidad. Probablemente al menos una persona por familia migraba temporalmente.

Los cambios en la alimentación familiar tanto en cantidad como en calidad fueron importantes, aunque más significativos respecto a la calidad, ya que los porcentajes de adecuación en varios nutrimentos mejoraron bastante, sobre todo en el consumo de energía y proteínas, como puede verse en el cuadro III. Las dietas de 1996 son mejores en calidad y cantidad aunque no están bien equilibradas: en algunos nutrimentos sobrepasan las recomendaciones y en otros siguen sien- do deficientes. La calidad proteica mejoró pues se registró un aumento en el consumo de alimentos de origen animal.

Se encontró una disminución en el aporte energético proveniente de cereales, con un incremento en el aporte derivado de frijoles, verduras, frutas y diversos alimentos industrializados. Aumentaron los consumos de colesterol y grasas saturadas pero, al mismo tiempo, disminuyó el consumo de fibra en las cuatro comunidades. Unicamente en Guía del Porvenir los cambios no fueron tan claros, quizá porque en esa comunidad desde el estudio de 1984 la dieta que se consumía era menos tradicional.

Los cambios dietéticos más importantes son la incorporación de alimentos industrializados, sobre todo de los baratos y altos en grasas, azúcares y sal, así como un aumento en las cantidades de alimentos de origen animal. El consumo de refrescos se elevó considerablemente y fue evidente la presencia de alimentos infantiles en los hogares. Se puede concluir que la alimentación de estas poblaciones comienza a seguir un patrón de dieta de tipo más urbano, con las ventajas y desventajas propias de esa situación. De acuerdo con lo anterior, la alimentación y el estado de nutrición de las cuatro comunidades mejoraron, a pesar de que habían pasado por cambios agrícolas importantes: primero, alrededor de 1970 se vieron obligadas a cambiar su agricultura tradicional de milpa, o sea de producción de maíz y algunos alimentos complementarios para autoconsumo, por el cultivo comercial de sorgo, y después, alrededor de 1990, sufrieron las consecuencias de una crisis comercial del mismo producto, pues tuvieron que competir con importaciones de forraje a precios muy bajos del mercado común norteamericano. A pesar de esto, tanto en la encuesta de 1984 como 
en la de 1996 hubo tres mejorías entre la población campesina de las cuatro comunidades tan diferentes: en el consumo total de energía, en la diversidad dietética y en el estado nutricional de los niños. Es importante mencionar, según se observa en el cuadro $\mathrm{V}$, que no todos los niños mejoraron pues, como se notifica en otro estudio hecho en la Chontalpa 25 años atrás, ${ }^{4}$ el sector más pobre y marginal permanece igual a pesar de los cambios sociales. Los resultados de 1996 muestran que donde más disminuyó la prevalencia de la desnutrición, de acuerdo con el indicador peso para la edad, fue en Bateas Alcalde, aumentando ligeramente la desnutrición grave. La desnutrición leve es la de mayor prevalencia en las cuatro comunidades, al tiempo que aumentaron las cifras de la categoría de sobrepeso, con excepción de Quebrantadero.

Respecto al indicador peso para la talla, los resultados muestran que la prevalencia de bajo peso en las cuatro comunidades pasó de 5 a 12\% en 1984, y de 9 a $16 \%$ en 1996. Este cambio también pudo deberse a un mejor crecimiento longitudinal y no necesariamente a un aumento de la desnutrición.

Ante la mejoría detectada en las cuatro comunidades ubicadas en distintas situaciones geográficas y, hasta cierto punto, culturales, se podría concluir que el cambio de la agricultura de maíz para autoconsumo a la producción comercial de sorgo, ha beneficiado el estado nutricional de las mismas. Empero, ésa no es la situación real, pues hubo muchos cambios de naturaleza económica y social. Es indudable que existe una relación entre el cambio agrícola y el social, aunque no necesariamente de causa-efecto, porque ambos pueden ser fenómenos simultáneos de origen común o independiente. Lo que se encontró en las cuatro comunidades fue que pasaron de una economía de autoabastecimiento a una economía comercial y que mejoraron en su alimentación y nutrición. La existencia de una relación de causa-efecto entre esos dos fenómenos es muy difícil de probar, pero es sostenida por unos autores y fuertemente combatida por otros que afirman lo contrario; estos últimos plantean que la economía tradicional protege el estado de nutrición y la salud comunales y que la comercial los agrede. ${ }^{15,16} \mathrm{De}$ hecho, en el mundo existe un equilibrio, tanto en experiencias como en opiniones, entre los investigadores que han encontrado o consideran que el rompimiento de los sistemas alimentarios tradicionales es sumamente negativo para los pueblos, y los que han encontrado o consideran lo contrario. Quizá esto se deba a que las experiencias han sido con poblaciones en diversa situación de preparación para el cambio o en condiciones socioeconómicas distintas.
La hipótesis de este estudio, cuyo planteamiento es que cambiar bruscamente el equilibrio tradicional de una comunidad, así como su sistema alimentario y su forma ancestral de cultivar, guardar, procesar y consumir el maíz y otros productos de la milpa la puede perjudicar gravemente, no fue probada, pero indudablemente tampoco se comprueba la hipótesis contraria. Eso significa que a pesar de los resultados positivos que se detectaron, no es posible generalizarlos porque, aunque la investigación fue desarrollada en condiciones geográficas diferentes, también se hizo en condiciones sociales parecidas, sobre todo en lo que concierne a lo que se podría llamar la preparación para el cambio. Desde los años sesenta, en todo el país se provocó un cambio que dejó de lado los cultivos tradicionales y favoreció el comercio agrícola protegiendo nuevos cultivos. Lo anterior se debió a una nueva demanda originada en el crecimiento de las ciudades y el desarrollo industrial, que requería de productos variados como algodón, azúcar, soya, sorgo, etcétera; por otra parte, el gobierno comenzó a estimular la exportación-importación de varios productos agrícolas, sobre todo en la relación comercial que sostiene con EUA, dentro del marco de una política denominada de "ventajas comparativas"; de acuerdo con ello cada país, según sus características climáticas, de suelos y de mano de obra, debe producir y exportar lo que considera apropiado. Ahora con el TLCAN este tipo de política agrícola se ha vuelto predominante.

Debido a las nuevas condiciones de la demanda, muchas comunidades tradicionales tuvieron que cambiar y, desafortunadamente, este estudio no registró lo que pasó al empezar el cambio, o sea 12 años antes de la primera encuesta. Probablemente en sus inicios el cambio fue traumático, pero hacia 1984 la situación ya se había estabilizado, con un consumo más variado, y para 1996 las comunidades demostraron su capacidad de adaptación a la crisis mediante la puesta en marcha de diversas estrategias de supervivencia.

Sin lugar a dudas debió de ser muy difícil para muchas comunidades rurales del país el hecho de que, en el curso de una misma generación, se cambiara desde una situación tradicional, aparentemente protegida por los mecanismos culturales de la familia y la comunidad, a una comercial que requiere una visión del trabajo diferente, con la adopción de diversas estrategias de sobrevivencia, sobre todo la emigración a un país tan distinto culturalmente como EUA; sin embargo, lo hicieron, conservando las ligas y los lazos familiares y comunitarios, al mismo tiempo que continúan haciendo producir su tierra, aunque en un contexto distinto al tradicional. 


\section{Referencias}

1.Von Braun J, Kennedy E. Commercialization of subsistence agriculture: Income and nutritional effects in developing countries.W ashington, D.C.: International Food Policy Research Institute, 1986:29-32.

2. DeW alt KM. N utritional strategies and agricultural change;Ann Arbor: UMI, Presearch Press, 1983:56.

3. 0 mawale B. Incorporating nutrition concerns into the specification of desired technology characteristics in international agricultural research. En: Pinstrop-Andersen et al, ed. International agricultural research and human nutrition. W ashington, D.C.: IFPRI, 1984:57-77.

4. Hernández M, Pérez-Hidalgo C, Ramírez J, Madrigal H, C hávez A. Effect of economic growth on nutrition in a tropical community. Ecol Food N utr 1974;3:283-291.

5. 0 kere LC. The anthropology of food in rural Igboland, Nigeria: Socioeconomic and cultural aspects of food and food habits in rural Igboland. Lanham (Md): University Press of A merica, 1983:125-143.

6. Barkin D, D e W alt BR, D e W alt KM, Escudero JC. El impacto nutricional de la substitución del cultivo de maíz por sorgo en 4 comunidades. En: A vila A, Barkin D, De W alt B, De W alt $M$ et al, ed. ¿Producir para la desnutrición? México, D.F.: Centro de Ecodesarrollo/Fundación Friedrich N eumann, 1988:59-86.

7. De W alt BR, De W alt KM, Escudero JC, Barkin D.Agrarian reform and small-farmer welfare:Evidence from four Mexican communities. Food $\mathrm{N}$ utr Bull 1987;9:46-52.
8. Menchú MT. Método de recordatario de un día. En: Madrigal H, Martínez $\mathrm{H}$, ed. Manual de encuestas de dieta. Cuernavaca, México: Instituto $\mathrm{N}$ acional de Salud Pública, Perspectivas en Salud Pública, núm. 23,1996;65-80.

9. Chávez $M, C$ hávez A, Roldán JA, Ledezma JA, Pérez-Gil S, Hernández $S L$ et al.Tablas de valor nutritivo de los alimentos de mayor consumo en México. México, D.F.: Pax, 1996.

10. Habicht JP. Estandarización de métodos epidemiológicos cuantitativos sobre el terreno. Bol 0 ficina Sanit Panam 1974;76:375-385.

11. O rganización Mundial de la Salud. Medición del cambio del estado nutricional. Ginebra: OMS, 1983.

12. Chávez A, Chávez M, Roldán A, Bermejo $S$, Avila A, Madrigal H. Food and nutrient consumption in rural areas. In:The food and nutrition situation in Mexico. México, D.F.: Pax, 1996:91-102.

13. Hernández $M$, A guirre J, Serrano L, Escobar M, Chávez A. La alimentación de obreros y sus familias. México, D.F.: Instituto $\mathrm{N}$ acional de la N utrición Salvador Zubirán/C O N ACYT, 1983:60.

14. Kershenobich D, Milke P. El alcohol: adicción y daño hepático. Cuadernos de Nutrición 1996; 19:6-14.

15. Suárez $B$, Barkin $D, D$ e W alt $B$, Hernández $M$, Rosales R. The nutritional impact of rural modernization: Strategies for smallholder survival in Mexico. Food N utr Bull 1987:9:30-35.

16. Dewey KG. Nutritional consequences of the transformation from subsistence to commercial agriculture in Tabasco. Human Ecology 1981; 9:151-187. 